\title{
Measurement of polarised distribution functions at HERMES
}

\author{
A. Fantoni ${ }^{a *}$ \\ aNFN - Laboratori Nazionali di Frascati, \\ Via E. Fermi, 40 - 00044 Frascati (Roma), Italy \\ e-mail: alessandra.fantoni@lnf.infn.it
}

HERMES is a second generation experiment to study the spin structure of the nucleon, in which measurements of the spin-dependent properties of semi-inclusive deep-inelastic lepton scattering are emphasized. By means of the flavor tagging technique, individual spin contributions have been determined directly from spin asymmetries of hadrons with the appropriate flavor content. Moreover, the first experimental results from measurements of single-spin asymmetries for pions and kaons in deep inelastic scattering with transverse target polarisation are presented.

\section{THE SPIN STRUCTURE OF THE NUCLEON}

Polarised deep-inelastic lepton scattering (DIS) has been the primary experimental basis for our present understanding of the spin structure of the nucleon. In a formal way, the nucleon's total spin of $1 / 2$ can be decomposed into its constituents:

$$
\begin{gathered}
<s_{N}>=\frac{1}{2}=\frac{1}{2} \Delta \Sigma+\Delta G+\Delta L= \\
\frac{1}{2}\left(\Delta u_{v}+\Delta d_{v}+\Delta q_{s}\right)+\Delta G+\Delta L_{q}+\Delta L_{G}
\end{gathered}
$$

where the three terms are the contributions from the quark and gluon spins and from their orbital angular momentum. The total quark helicity $\Delta \Sigma$ is defined as the sum of the helicity distributions of up, down and strange quarks and antiquarks. The HERMES experiment can probe all the single pieces of this relation. In fact, from inclusive DIS measurements it is possible to obtain the polarised structure function $g_{1}$, related to $\Delta \Sigma$; from semi-inclusive DIS (SIDIS) measurements with observation of the produced hadron, the helicity distributions of individual quark flavors in the nucleon can be extracted; by measuring the asymmetry for DIS of a transversely polarised target it is possible to access the transversity distribution, which probes the spin of the nucleon while switching off the gluon contribution; moreover,

*on behalf of the HERMES Collaboration. the possible role of quark orbital angular momentum is addressed by studying (almost) exclusive reactions, which can be interpreted in terms of the Generalized Parton Distributions (GPDs).

\section{THE HERMES EXPERIMENT}

HERMES is located in the East Hall of the HERA $e p$ collider at DESY in Hamburg, Germany and it has taking data since 1995, immediately after its installation. HERMES scatters longitudinally polarised electron and positron beams of $27.5 \mathrm{GeV}$ from longitudinally or transversely polarised targets internal to the HERA storage ring. The HERMES spectrometer is described in detail in [1]. It is a forward spectrometer in which both scattered positron and produced hadrons are detected within an angular acceptance of \pm 170 mrad horizontally and \pm (40-140) mrad vertically. The beam line separates the spectrometer in an upper and lower part with independent detector systems and trigger for the top and bottom sections. Drift chambers before and after the spectrometer magnet are used for the track reconstruction and momentum analysis. The Particle Identification (PID) is obtained by a transition radiation detector, an electromagnetic calorimeter with a preshower detector preceding it and a threshold Cherenkov counter, upgraded to a dual Ring Imaging System (RICH) in 1998: the use 
of these detectors in combination allows the identification of leptons with an efficiency of $98 \%$ or better with a hadron contamination of less than $1 \%$. The RICH provides full separation between charged pions, kaons and protons over essentially the entire momentum range of the experiment: this is a mandatory prerequisite in flavor tagging, which is the technique used to relate a produced hadron of a given type to a struck quark of a given flavor.

\section{SEMI-INCLUSIVE DIS: THE FLA- VOR DECOMPOSITION}

Semi-inclusive DIS (SIDIS) is a powerful tool to determine the separate contributions $\Delta q_{f}(x)$ of the quarks and antiquarks of flavor $f$ to the total spin of the nucleon. The hadron asymmetries $A_{1}^{h}(x)$ are related to the quark distributions $\Delta q_{f}(x)$ through the so-called purity matrix $P_{f}^{h}$, which describes the probability that a hadron $h$ originates from a quark of flavor $f$. They have been extracted using Monte Carlo simulations with the limited acceptance of the spectrometer taken into account. For the unpolarised quark distributions the CTEQ5L parameterization was used, for the fragmentation functions the parameters of the LUND string fragmentation model implemented in JETSET have been tuned to fit the hadron multiplicities measured. The quark helicity distributions $\Delta q(x)_{f}(x)$ can be derived by multiplying each of the quark polarisations with the corresponding unpolarised parton distribution function at fixed $Q^{2}=2.5 \mathrm{GeV}^{2}$. For the first time, a global analysis of the inclusive and semi-inclusive spin asymmetries for $\pi^{+}, \pi^{-}$, $K^{+}$and $K^{-}$with smearing effects unfolded has been carried out for longitudinally polarised targets of hydrogen and deuterium [2]. The results of the decomposition are presented in Fig.1 and compared to LO QCD analyses. No assumptions have been made on the symmetry of the sea flavors, except that $\Delta \bar{s} / \bar{s}$ is assumed to be zero. For $x>0.3$, the polarisation of the sea flavors was fixed to zero and the small variations from this value have been included in the systematic errors. As expected, the helicity density of the $u$ quark is found to be positive and large at $x>0.1$, and that of the $d$ quark is negative, while the helicity densities of the light sea quarks are found to be compatible with zero. For sea quarks there is no disagreement with the QCD fits within the experimental uncertainties. Moreover, a precise "isoscalar" extraction of the total strange quark helicity density $\Delta S=\Delta s+\Delta(\bar{s})$ has been extracted from polarisation measurements in scattering on the deuteron alone, which is an isoscalar target.

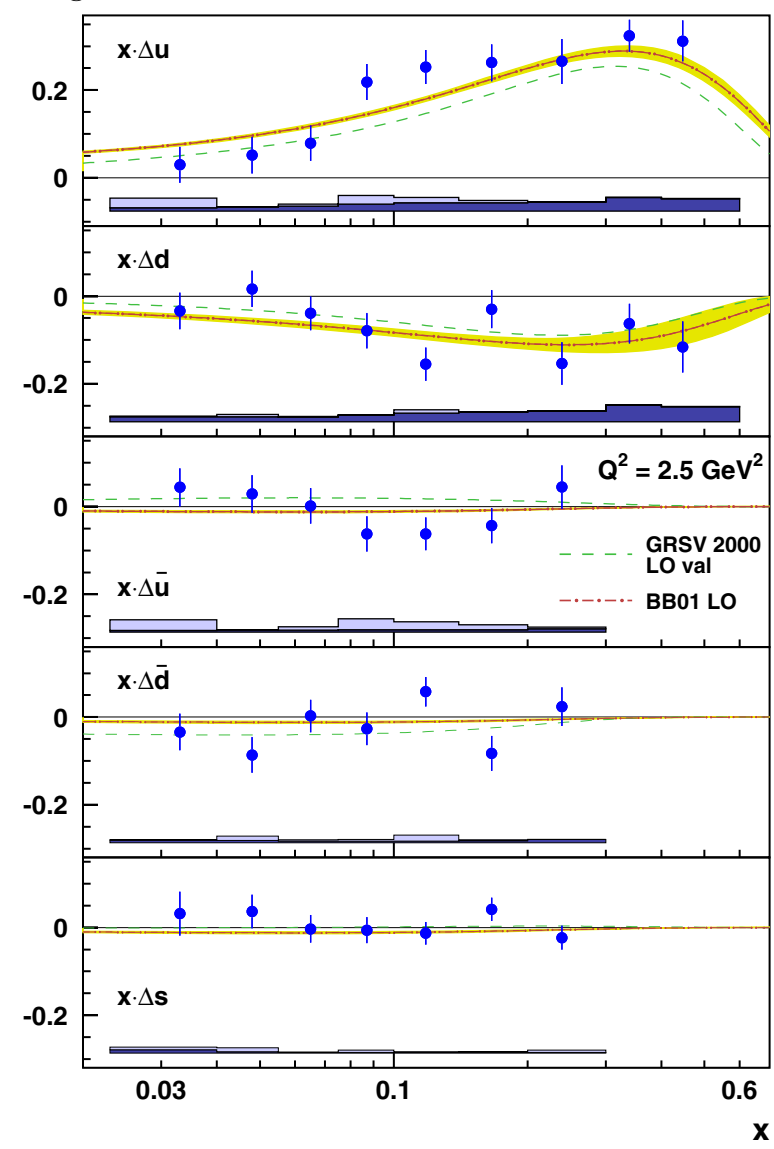

Figure 1. The $x$-weighted polarised quark densities. The plots show a five parameter fit to the data assuming a symmetric strange sea polarisation. The data have been evolved to a common value of $Q_{0}^{2}=2.5 \mathrm{GeV}^{2}$. The curves show $\mathrm{LO}$ QCD analyses fits to the inclusive world polarised data set: the dashed one is the GRSV2000 parameterization [3], and the dashed-dotted curve is an alternate parameterization of Blümlein and Böttcher [4]. 


\section{TRANSVERSE SPIN ASYMME- TRIES}

Apart from the structure functions $F_{1}(x)$ and $g_{1}(x)$, there is a third leading order structure function $h_{1}(x)$, known as transversity distribution, which is required to provide a complete description of the quark structure of the nucleon. It is related to a forward scattering amplitude involving helicity flip of both quark and target nucleon. However, in a basis of transverse eigenstates it becomes a number density reflecting the difference in probabilities to find quarks in a transversely polarised nucleon with their spin aligned to the spin of the nucleon and quarks with their spin anti-aligned. Since hard interactions conserve chirality, transversity has so far remained unmeasured in inclusive processes due to its chiral-odd nature. Contrary to the longitudinal case, this distribution can only be accessed in SIDIS processes as both the quark and nucleon need to flip their spin. Hence, additional angular momentum must be absorbed by a hadron produced in the reaction. The transverse polarisation of the struck quark can influence the transverse momentum component of the produced hadron orthogonal to the virtual photon, thereby leading to an azimuthal angle $\phi$ dependence of the cross section about the virtual photon direction in the lepton scattering plane. A fragmentation function (FF) describing this spinmomentum correlation is known as the Collins FF $H_{1}^{\perp}(z)$ : it represents the interference of two scattering amplitudes with different imaginary parts giving rise to single-spin asymmetries. Azimuthal spin asymmetries can also be generated by the $\mathrm{T}$ odd Sivers distribution function (DF) $f_{1 T}^{\perp}\left(x, k_{T}\right)$ that appears in the cross section together with the unpolarised FF $D(z)$. The $f_{1 T}^{\perp}\left(x, k_{T}\right)$ describes a correlation between the transverse polarisation of the target nucleon and the intrinsic transverse momentum of the struck quark. The Collins and Sivers mechanisms can be separated in a transversely polarised target since they have a different azimuthal dependence with respect to the axis of transverse polarisation. While the Sivers mechanism give rise to a $\sin \left(\phi-\phi_{S}\right)$ behavior, the Collins mechanism exhibits a $\sin \left(\phi+\phi_{S}\right)$ be- haviour in the azimuthal angle, with $\phi$ being the azimuthal angle between the hadron production plane and the lepton scattering plane and $\phi_{S}$ the angle between the lepton scattering plane and the transverse spin component of the target nucleon. The first measurement of non-zero Collins and Sivers moments for charged pions as a function of $x, z$ and transverse hadron momentum $P_{h \perp}$ has been recently published by HERMES [5]. The Collins amplitude is positive for $\pi^{+}$and negative for $\pi^{-}$. These opposite signs can be expected if the transversity densities resemble the helicity densities, although the absolute magnitude of the $\pi^{-}$asymmetry is surprising, suggesting a substantial disfavoured Collins function with opposite sign to that of the favoured function. The Sivers amplitude is significantly positive for $\pi^{+}$, providing an indication for nonzero orbital angular momentum of quarks in the nucleon and the first evidence of a T-odd DF, while it is consistent with zero for $\pi^{-}$. The apparently negative value of the naively T-odd Sivers DF describing this correlation can in future be compared to its appearance in polarised Drell Yan processes, where it is predicted to have opposite signs due to its fundamental time reversal invariance in QCD. For the first time, these moments have been extracted also for the charged kaons, using a maximum likelihood based fit: the results are shown in Fig.2. Effects of acceptance, instrumental smearing and QED radiation were all found to be negligible in Monte Carlo simulations and the largest contribution to the systematic uncertainties is due to the target polarisation. While there is no reason to expect a similar amplitude for $K^{-}$and $\pi^{-}$, being a $K^{-}$a fully sea object, the $u$-quark dominance in DIS would suggest a similar amplitude for $K^{+}$ and $\pi^{+}$. However a smaller amplitude is observed for $K^{+}$than for $\pi^{+}$. As in the case of unpolarised fragmentation functions, the Collins function may thus differ for fragmentation of $u$ into $K^{+}$and $u$ into $\pi^{+}$. On the other hand, the amplitude of Sivers moment for $K^{+}$is roughly twice as big that for $\pi^{+}$in the region $x \approx 0.1$. This suggests that the sea quarks may provide an important contribution to the Sivers function and thus may carry significant orbital angular momentum in the nucleon. 


\section{SUMMARY AND OUTLOOK}

Experiments studying the structure of the nucleon have expanded considerably beyond the well-known inclusive deep-inelastic lepton scattering experiment, which have yielded very precise data on the unpolarised structure function $F_{2}(x)$ and a good knowledge of the polarised structure function $g_{1}(x)$. However, in spite of many years of experiments, a detailed decomposition of the spin of the nucleon remains still elusive. HERMES has obtained impressive results on this field, trying to solve some of the central questions of the so-called "spin puzzle". For the first time, the independent determination of five of six quarks polarisations in the nucleon have been obtained, by using a longitudinally polarised atomic hydrogen and deuterium gas targets. Moreover, the first measurement of asymmetries directly related to transversity and the Sivers function have been made. The Collins and Sivers azimuthal moments are extracted for charged pions and also for charged kaons. The preliminary results reported here will be improved with the inclusion of the 2005 data, which will double the statistic, presented. The results to date provide a demonstration of a new tool for studying the transverse structure of the nucleon.

\section{REFERENCES}

1. HERMES Collab. (K. Ackerstaff et al.), Nucl. Instr. and Meth. A 417 (1998) 230.

2. HERMES Collab. (A. Airapetian et al.), Phys. Rev. Lett. 92 (2004), 012005; HERMES Collab. (A. Airapetian et al.), Phys. Rev. D 71 (2005) 012003.

3. M. Glück, E. Reya, M. Stratmann, and W. Vogelsang, Phys. Rev. D 63 (2001) 094005 .

4. J. Blümlein and H. Böttcher, Nucl. Phys. B 636 (2002) 225.

5. HERMES Collab. (A. Airapetian et al.), Phys. Rev. Lett. 94 (2005) 012002.
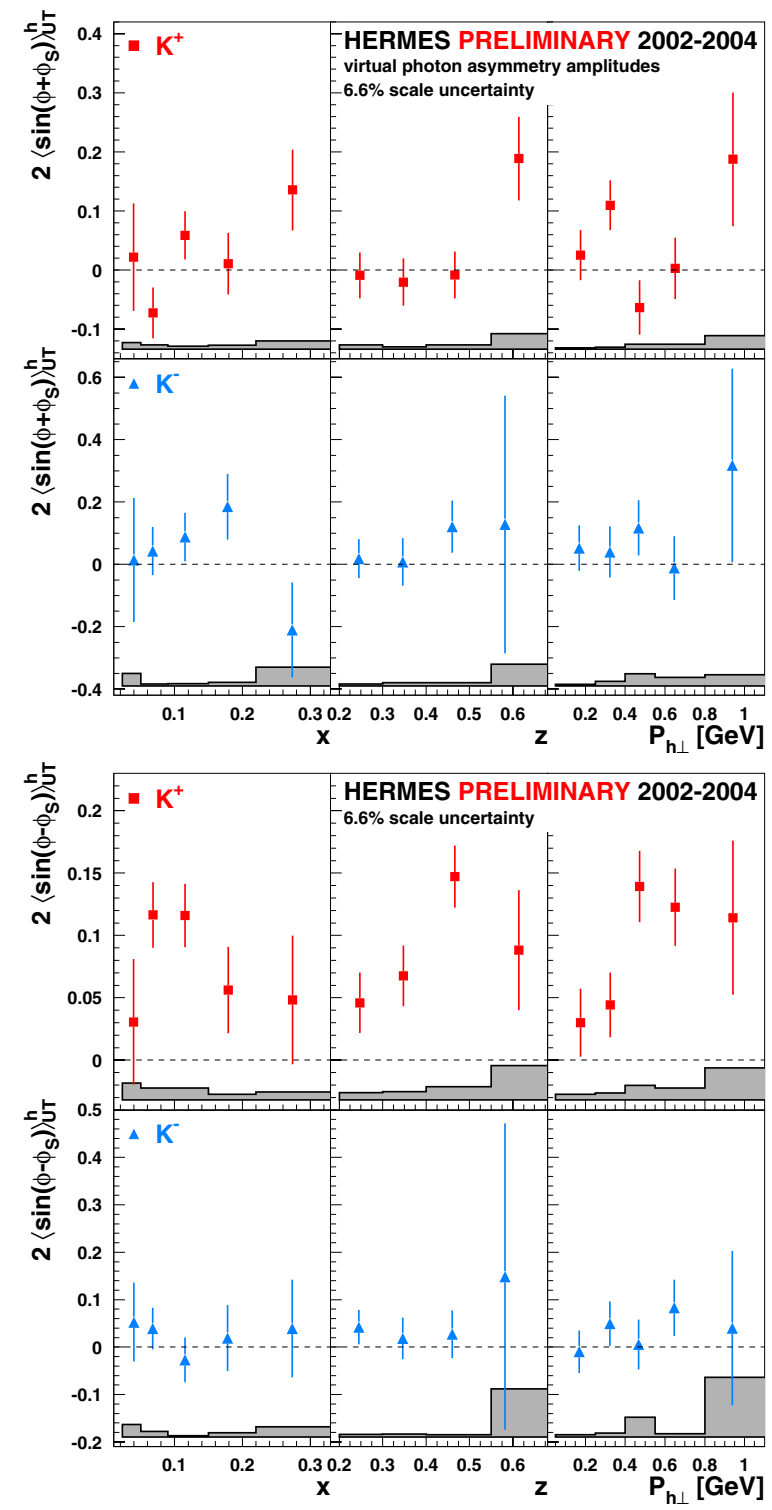

Figure 2. Virtual photon Collins (top) and Sivers (bottom) moments for charged kaons as a function of $x, z$ and transverse hadron momentum $P_{h \perp}$. Error bars represent the statistical uncertainties, while the lower band represents the maximal systematic uncertainty due to acceptance and detector smearing as well as possible contributions from $\cos \phi$ moments of the unpolarised cross section. The $6.6 \%$ scale uncertainty is due to the target polarisation. 